\title{
Identification and characterization of zebrafish Tlr4 co-receptor Md-2
}

${ }^{1}$ Institute of Molecular Biology, ${ }^{2}$ Institute of Neuroscience, ${ }^{3}$ Department of Chemistry and

11 Biochemistry, ${ }^{4}$ Department of Biology, University of Oregon, Eugene, OR 97403, ${ }^{5}$ Humans and the Microbiome Program, CIFAR, Toronto, Ontario M5G 1Z8, Canada

\section{Funding sources:}

15 This research was funded by grants from the American Heart Association (AHA-

16 15BGIA22830013, MJH) and the National Institutes of Health (NIH-T32GM007413, ANL,

17 NIH-F32DK107318, MNH, P50GM09891, KG, NIH-R24OD026591, ACM). MJH is a Pew

18 Scholar in the Biomedical Sciences, supported by The Pew Charitable Trusts. The funders had

19 no role in study design, data collection and analysis, decision to publish, or preparation of the

20 manuscript. 


\section{SUPPLEMENTAL MATERIAL}

24 Fig S1: Alignment of Md-2 proteins from amphibians and various fishes

25 Fig S2: Human and zebrafish gene context BLAST hits on bamboo shark chromosomes

26 Fig S3: Human and zebrafish gene context BLAST hits on human chromosomes

27 Fig S4: Human and zebrafish gene context BLAST hits on chicken chromosomes

28 Fig S5: Human and zebrafish gene context BLAST hits on frog chromosomes

29 Fig S6: Human and zebrafish gene context BLAST hits on gar chromosomes

30 Fig S7: Human and zebrafish gene context BLAST hits on bonytongue chromosomes

31 Fig S8: Human and zebrafish gene context BLAST hits on catfish chromosomes

32 Fig S9: Human and zebrafish gene context BLAST hits on zebrafish chromosomes

33 Fig S10: Human and zebrafish gene context BLAST hits on pike chromosomes

34 Fig S11: Human and zebrafish gene context BLAST hits on cod chromosomes

35 Fig S12: Human and zebrafish gene context BLAST hits on puffer chromosomes

36 Fig S13: Comparison of zebrafish Tlr4a sequence used in this paper versus previous work

Table S1: Gene locations for ly96 synteny analysis

39 Table S2: Predicted zebrafish ly96 mutant gene products

40 Table S3: Genomes used for tlr4 synteny analysis

41 Table S4: Genes used to BLAST for human vs. zebrafish Tlr4 genomic context

File S1: Spreadsheet containing accession numbers and aligned sequences for Md-1 and Md-2 sequences. 
ABSTRACT

The zebrafish (Danio rerio) is a powerful model organism for studies of the innate

52 immune system. One apparent difference between human and zebrafish innate immunity is the

53 cellular machinery for LPS-sensing. In amniotes, the protein complex formed by Toll-like

54 receptor 4 and myeloid differentiation factor 2 (Tlr4/Md-2) recognizes the bacterial molecule

55 lipopolysaccharide (LPS) and triggers an inflammatory response. It is believed that zebrafish

56 have neither Md-2 nor Tlr4: Md-2 has not been identified outside of amniotes, while the

57 zebrafish $t$ lr4 genes appear to be paralogs, not orthologs, of amniote $T L R 4 s$. We revisited these

58 conclusions. We identified a zebrafish gene encoding Md-2, ly96. Using single-cell RNA-Seq,

59 we found that $l y 96$ is transcribed in cells that also transcribe genes diagnostic for innate immune

60 cells, including the zebrafish tlr4-like genes. Unlike amniote $L Y 96$, zebrafish ly96 expression is

61 restricted to a small number of macrophage-like cells. In a functional assay, zebrafish Md-2 and

62 Tlr4a form a complex that activates NF- $\mathrm{B}$ signaling in response to LPS, but ly96 loss-of-

63 function mutations gave little protection against LPS-toxicity in larval zebrafish. Finally, by

64 analyzing the genomic context of $t \operatorname{tr} 4$ genes in eleven jawed vertebrates, we found that $t \operatorname{lr} 4$ arose

65 prior to the divergence of teleosts and tetrapods. Thus, an LPS-sensitive Tlr4/Md-2 complex is

66 likely an ancestral feature shared by mammals and zebrafish, rather than a de novo invention on

67 the tetrapod lineage. We hypothesize that zebrafish retain an ancestral, low-sensitivity Tlr4/Md-2

68 complex that confers LPS-responsiveness to a specific subset of innate immune cells. 


\section{INTRODUCTION}

72 Amniote innate immune systems are exquisitely sensitive to lipopolysaccharide (LPS), a

73 component of the cell wall in Gram-negative bacteria (1-3). LPS is sensed by a protein complex

74 composed of Toll-like receptor 4 (Tlr4) and Md-2 (also known as LY96 and ESOP-1) (1, 4). LPS

75 binds in a pocket of Md-2, triggering dimerization of the Tlr4/Md-2 complex (Fig 1) (5). This, in

76 turn, activates a Myd88-dependent NF- $\kappa$ B response (6). When properly regulated, the LPS

77 activation of Tlr4/Md-2 regulates microbiome populations (7), recruits neutrophils to sites of

78 infection (8), and induces angiogenesis (9). When dysregulated, Tlr4/Md-2 activity induces

79 septic shock $(10,11)$, plays roles in inflammatory disorders $(11,12)$, and is a key player in the

80 tissue remodeling that accompanies tumorigenesis $(13,14)$.

81 The role of Tlr4/Md-2 in LPS-sensing outside of amniotes remains poorly understood.

82 Understanding this response in zebrafish (Danio rerio) is of particular interest, as the zebrafish is

83 a powerful model organism for studies of vertebrate innate immunity (15). Zebrafish have

84 mature genetic resources, rapid generation time, clear embryos, and facile germ-free derivation

$85(16,17)$. The zebrafish is increasingly popular as a model for understanding host-microbe

86 interactions (18), as well as a tool to understand the development of the innate immune system

87 (16). 


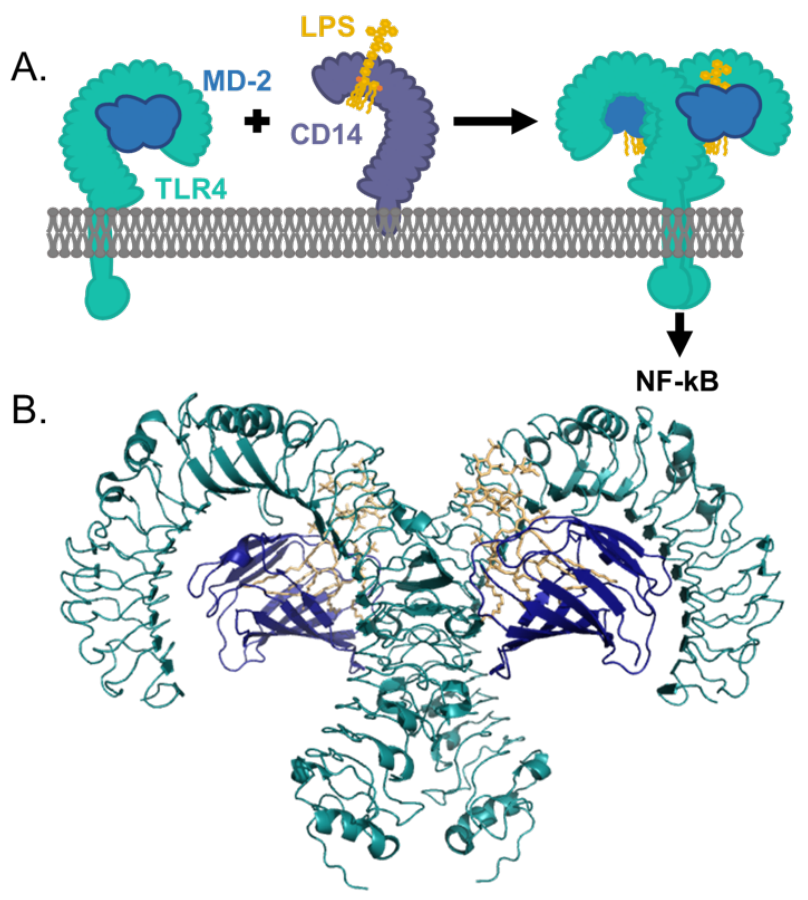

Fig 1. LPS activation of amniote Tlr4 requires cofactors Md-2 and Cd14. A) Schematic representation of LPS transfer from Cd14 to the Tlr4/Md-2 complex. LPS (yellow) is brought by Cd14 (purple) and loaded into Md-2 (navy blue). Md-2 is bound by Tlr4 (cyan). Binding of LPS to the Md-2 co-receptor causes dimerization of the Tlr4/Md-2 complex, activating a downstream inflammatory response. B) The interface between human Tlr4 (cyan) and Md-2 (navy blue) is extensive. Both are required to form a productive interaction with LPS (yellow). Structure shown was made from PDB 3FXI (19). amniotes. As in amniotes, LPS triggers Myd88-dependent NF- $\kappa$ B inflammatory response (22, 23). Further, the expression patterns of genes induced by LPS stimulation are highly similar between mouse and zebrafish (24). There are, however, several lines of evidence that suggest

101 Tlr4/Md-2 is not involved. Most critically, the gene encoding the essential co-receptor Md-2 has

102 not been identified in zebrafish and other ray-finned fishes $(20,21,25)$. Further, zebrafish Tlr4

103 proteins do not activate NF- $\kappa \mathrm{B}$ in response to LPS in ex vivo assays, even when complemented

104 with a mouse or human Md-2 (20,21). Finally, zebrafish do not have a direct ortholog to

105 amniote $t l r 4$. Rather, they possess three tlr4-like genes — tlr4ba, tlr4bb, and tlr4al—that are 
thought to have arisen from an ancestral Toll-like receptor lost in tetrapods but retained in ray-

107 finned fishes (21). These observations have led to the hypothesis that zebrafish respond to LPS

108 by a non-Tlr4/Md-2-dependent pathway.

We set out to carefully revisit these conclusions using resources unavailable when the

110 initial investigations of zebrafish Tlr4 were performed. Using careful bioinformatics, we found

111 an ortholog of the gene encoding Md-2 (ly96) in zebrafish and other ray-finned fishes. When co-

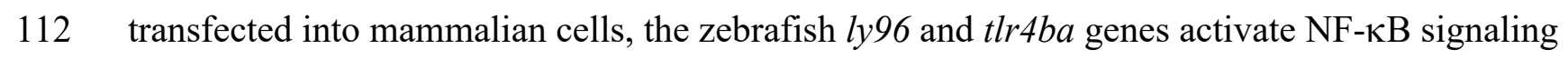

113 in response to LPS. Single-cell RNA-seq experiments on larval zebrafish revealed that the gene

114 is expressed in a small subset of cells that express the zebrafish tlr4-like genes and the

115 macrophage-specific gene mpeg1.1 (26). This contrasts with amniotes, in which TLR4 and LY96

116 are both broadly expressed (27). Further, unlike mammalian TLR4 and LY96 mutants that

117 exhibit increased resistance to systemic LPS challenge $(4,28)$, zebrafish larvae with loss of

118 function $l y 96$ mutations are not protected from LPS toxicity. Finally, we revisited the history of

119 the tlr4 gene in zebrafish, finding that formation of an LPS-sensitive Tlr4/Md-2 complex is

120 likely an ancestral feature shared by mammals and zebrafish, rather than a de novo invention on

121 the tetrapod lineage. We hypothesize that zebrafish preserve an ancestral, low-sensitivity

122 Tlr4/Md-2 complex that plays an LPS-sensing role in a small population of innate immune cells.

124 MATERIALS \& METHODS

125 Phylogenetic reconstruction analysis

126 We constructed curated databases of Md-1, Md-2, Tlr4, and Cd180 protein sequences

127 from across the vertebrates. Cd180 and Md-1 are paralogs of Tlr4 and Md-2, respectively (29).

128 We obtained amino acid sequences of these proteins from NCBI, Ensembl, Fish1TK, amphibian

129 transcriptomes (30-33), UniProt, and ZFIN. We constructed a multiple sequence alignment for 
130 Tlr4 and Cd180 and for Md-2 and Md-1 using MSAPROBS (34), followed by manual editing in

131 MEGA (35). We trimmed the alignment to remove highly variable (and therefore unalignable)

132 regions. We used PHYML $(36,37)$ with subtree pruning and re-grafting to construct the ML

133 phylogeny. Pilot analyses revealed that the JTT substitution model with 8 rate categories and a

134 floating gamma distribution parameter yielded the highest likelihood trees (38-40). An Akaike

135 information criterion (AIC) test was used to control for overfitting (41). We rooted our trees at

136 the duplication of these proteins in early vertebrates. Alignment figures in supplement were

137 made with JalView (42).

\section{Synteny analysis}

$140 \quad$ For the $l y 96$ synteny analysis, we used the Ensembl synteny module (43) to map

141 homologs onto the chromosomes of species of interest. For the tlr4 synteny analysis, we took the

14222 genes flanking human TLR4 (11 on each side) and the 22 genes flanking zebrafish $t$ tr4. We

143 used tblastn with default parameters to BLAST these sequences against 11 vertebrate genomes.

144 We discarded all hits with e-value $<0.001$ and then calculated a running average of the $\log (\mathrm{e}-$

145 value) along each chromosome with a sliding window of 10,000 bases. Finally, we divided this

146 running average by the maximum observed log (e-value)/bp value across all genomes. This

147 value occurs for the window centered on the zebrafish $t$ tr 4 gene. On the final relative scale, 0.0

148 indicates no hits observed in a given window and 1.0 is the maximum e-value per base pair. The

149 complete analysis pipeline is implemented in a collection of shell scripts and jupyter notebooks

150 (https://github.com/harmslab/vertebrate-tlr4-synteny/). 


\section{Gene expression analysis}

Whole 6 days post fertilization (dpf) zebrafish were euthanized by tricaine methane

155 sulfonate overdose, flash frozen in $1 \mathrm{~mL}$ of Trizol (Ambion), thawed, and homogenized.

156 Chloroform $(200 \mu \mathrm{L})$ was added to each tube followed by mixing, centrifugation at $12,000 \mathrm{~g}$ for

15710 minutes at $4^{\circ} \mathrm{C}$, transfer of the aqueous phase to a separate tube, addition of $200 \mu \mathrm{L}$ ethanol,

158 and binding of sample to an RNeasy mini kit column (Qiagen). RNA was washed and eluted

159 according to the manufacturer's instructions and treated with RQ1 DNase (Promega). RNA was

160 reverse transcribed into cDNA using Superscript II Reverse Transcriptase (Invitrogen) and an

161 oligo dT (20) primer, then amplified by PCR using gene-specific primers for zebrafish ly96 (5' -

162 TGTATGGCATCTGAGAAAGCAGA - 3' and 5' - AAGAGCAGGGGGAAACAGTC - 3') and

163 the housekeeping gene $b 2 m\left(5^{\prime}\right.$ - ACGCTGCAGGTATATTCATC - 3' and 5' -

164 TCTCCATTGAACTGCTGAAG - 3' ). PCR products were separated by electrophoresis on a 6\%

165 bis-Acrylamide (19:1) gel that was stained with 1X SYBR green 1 nucleic acid gel stain

166 (Invitrogen) and imaged using an AlphaImagerHP (Alpha Innotech). The identity of the ly96 RT-

167 PCR product was verified by Sanger sequencing.

169 Single-cell RNA-Seq

170 Single-cell analysis of transcription patterns of $l y 96, t \operatorname{tr} 4 b a, t \operatorname{tr} 4 b b$, and $t \operatorname{lr} 4 a 1$ was performed

171 using the recently released Zebrafish Single-Cell Transcriptome Atlas (44). Briefly, dissociated

172 cells were run on a 10X Chromium platform using v2 chemistry. Dissociated samples for each

173 time point (1,2 and $5 \mathrm{dpf})$ were submitted in duplicate to determine technical reproducibility.

174 The resulting cDNA libraries were sequenced on either an Illumina Hi-seq or an Illumina Next-

175 seq. The resulting sequencing data were analyzed using the $10 \mathrm{X}$ Cellranger pipeline, version 
2.2.0 (45) and the Seurat software package for $\mathrm{R}, \mathrm{v} 3.4 .4(46,47)$ using standard quality control, normalization, and analysis steps. We aligned reads to the zebrafish genome, GRCz11_93, and counted expression of protein coding reads. The resulting matrices were read into Seurat where we performed PCA and UMAP analysis on the resulting dataset with 178 dimensions and a resolution of 13.0, which produced 220 clusters and one singleton. Differential gene expression analysis was performed using the FindAllMarkers function in Seurat and Wilcoxon rank sum test.

\section{Cell Culture and Transfection Conditions}

Mammalian expression vectors containing human TLR4 and mouse Tlr4 were obtained

186 from Addgene (\#13085 and \#13086), originally deposited by Ruslan Medzhitov. Human CD14

187 and ELAM-Luc were also obtained from Addgene (\#13645 and \#13029) originally deposited by

188 Doug Golenbock. Human MD-2 was obtained from the DNASU Repository (HsCD00439889)

189 and contains a C-terminal V5-tag. Mouse Md-2 (UniProt \#Q9JHF9) and Cd14 (UniProt

190 \#P10810), as well as opossum Md-2 (UniProt \#F6QBE6), Cd14 (NCBI Accession

191 \#XP_007473804.1) and chicken Md-2 (UniProt \#A0A1D5NZX9), and Cd14 (UniProt

192 \#B0BL87) were designed to be free of restriction sites, codon-optimized for human expression,

193 and purchased as mammalian expression vector constructs in pcDNA3.1 (+) from Genscript

194 (New Jersey, USA). Zebrafish $t l r 4 b a$ and $l y 96$ were also obtained from Genscript in pcDNA3.1

$195(+)$. Zebrafish $t l r 4 b b$ was a gift from Carol Kim. We re-cloned this protein from its original

196 vector into pcDNA3.1 (+) to limit variability in expression due to differences in vector size and 197 promoter. 

transfection, a confluent $100 \mathrm{~mm}$ plate of HEK293T/17 cells was treated at room temperature with $0.25 \%$ Trypsin-EDTA in HBSS and resuspended with an addition of DMEM $+10 \%$ FBS.

202 This was diluted 4-fold into fresh medium and $135 \mu \mathrm{L}$ aliquots of resuspended cells were 203 transferred to a 96-well cell culture treated plate. Transfection mixes were made with $10 \mathrm{ng}$ of $204 \mathrm{tlr} 4,1 \mathrm{ng}$ of $c d 14,10 \mathrm{ng}$ of ly96, $1 \mathrm{ng}$ of Renilla, $20 \mathrm{ng}$ of ELAM-Luc, and $58 \mathrm{ng}$ of pcDNA3.1

205 (+) per well for a total of $100 \mathrm{ng}$ of DNA, diluted in OptiMEM to a volume of $10 \mu \mathrm{L} /$ well. To the 206 DNA mix, $0.5 \mu \mathrm{L}$ per well of PLUS reagent was added followed by a brief vortex and room 207 temperature incubation for $10 \mathrm{~min}$. Lipofectamine was diluted $0.5 \mu \mathrm{L}$ into $9.5 \mu \mathrm{L}$ OptiMEM per 208 well. This was added to the DNA + PLUS mix, vortexed briefly and incubated at RT for 15 min. 209 The transfection mix was diluted to $65 \mu \mathrm{L} /$ well in OptiMEM and aliquoted onto a plate. Cells 210 were incubated with transfection mix overnight (20-22 hrs) and then treated with LPS. E. coli K21112 lipopolysaccharide (LPS) (tlrl-eklps, Invivogen) was dissolved at $5 \mathrm{mg} / \mathrm{mL}$ in endotoxin-free 212 water, and aliquots were stored at $-20^{\circ} \mathrm{C}$. To avoid freeze-thaw cycles, working stocks of LPS 213 were prepared at $10 \mu \mathrm{g} / \mathrm{mL}$ and stored at $4^{\circ} \mathrm{C}$. To prepare treatments, LPS was diluted in $25 \%$ 214 phosphate buffered saline and 75\% DMEM. Cells were incubated with treatments for $4 \mathrm{hr}$. The 215 Dual-Glo Luciferase Assay System (Promega) was used to assay Firefly and Renilla luciferase

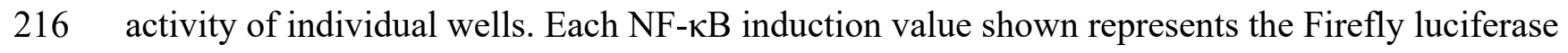
217 activity/Renilla luciferase activity, normalized to the buffer treated transfection control to 218 compare fold-change in NF- $\mathrm{BB}$ activation for treatments. 


\section{Generation of mutant zebrafish}

Zebrafish experiments were approved by the University of Oregon Institutional Animal

223 Care and Use Committee. Chop Chop (http://chopchop.cbu.uib.no) was used to design a guide

224 RNA (gRNA) targeting the first exon of zebrafish ly96 (si:dkey-82k12.13, GRCz11). A gRNA

225 template was generated by a template-free Phusion polymerase (New England Biolabs) PCR

226 reaction using a scaffold primer (5'-

GATCCGCACCGACTCGGTGCCACTTTTTCAAGTTGATAACGGACTAGCCTTATTTTA

ACTTGCTATTTCTAGCTCTAAAAC-3') and an ly96-specific primer (5'-

AGC-3'), then cleaned using the QIAquick PCR Purification Kit (Qiagen), transcribed using a

MEGAscript kit (Ambion), and purified by phenol-chloroform extraction and isopropanol

232 precipitation. Cas9 RNA was made by linearizing the pT3TS-nls-zCas9-nls plasmid (41) with

233 XbaI, purifying it using the QIAquick Gel Extraction Kit (Qiagen), performing an in vitro

234 transcription reaction using the T3 mMESSAGE kit (Invitrogen), and purifying the RNA using

235 the RNeasy Mini kit (Qiagen). AB strain zebrafish embryos were microinjected at the one cell

236 stage with 1-2 nL of a mixture containing $100 \mathrm{ng} / \mu \mathrm{L}$ Cas $9 \mathrm{mRNA}, 50 \mathrm{ng} / \mu \mathrm{L}$ gRNA, and phenol

237 red, and raised to adulthood. Fin DNA was amplified by PCR using primers specific to the

238 targeted region (5'- CAAATTGGATTCACAACAGAGC -3' and 5' -

239 CCATGGAAAATCAATGAAAAGC - 3'). Mosaic mutants were identified based on loss of an

240 HaeII restriction site and were outcrossed to wildtype AB zebrafish to generate heterozygotes.

241 Fish with loss-of-function mutations were identified by Sanger sequencing and further crossed to

242 generate three independent homozygous ly96 mutant lines. 


\section{Fish LPS survival assay}

WT and homozygous $l y 96$ mutant zebrafish embryos were grown under standard

246 conditions in separate $10 \mathrm{~cm}$ petri dishes at a density of one fish per $\mathrm{mL}$ of embryo medium

247 (EM), with fifty fish total per dish. At 5 dpf, lipopolysaccharides (LPS) purified from

248 Escherichia coli 0111:B4 (Sigma L2630) was dissolved in EM and added to dishes at a final

249 concentration of $0.6 \mathrm{mg} / \mathrm{mL}$, and control fish were mock treated with EM alone. Dead larvae, as

250 determined by lack of heartbeat, were counted and removed at regular intervals from 16 to 48

251 hours or from 16 to 72 hours after addition of LPS, at which time the experiment was terminated

252 and surviving fish were humanely euthanized.

\section{RESULTS}

\section{Zebrafish have a gene encoding MD-2}

The strongest evidence against Tlr4/Md-2 performing LPS-sensing in zebrafish is the

257 lack of Md-2. Md-2 is essential for LPS recognition by amniote Tlr4, as it contains the LPS

258 binding pocket (Fig 1). We therefore asked whether we could find a gene encoding Md-2 in bony

259 fishes.

260 We started by using the human MD-2 protein sequence to BLAST against the zebrafish

261 genome and transcriptomes. This returned no significant hits, so we took a more

262 phylogenetically informed strategy. Relative to humans, the earliest branching, functionally

263 characterized Tlr4/Md-2 complex is from chicken (Gallus gallus). We therefore "walked out"

264 from amniotes towards fishes, starting with amphibians. We BLASTed the human MD-2 protein

265 sequence against the Xenopus laevis genome. This revealed a hit to a hypothetical protein with

$26630 \%$ identity (OCT74818.1). When reverse-BLASTed against the human proteome, this hit 
267 returned Md-2. To validate the amino acid sequence, we compared it to the sequences of

268 functionally characterized Md-2 proteins from amniotes. We found that the $X$. laevis gene

269 appeared to be N-terminally truncated. Using XenBase, we identified the full-length transcript in

270 the transcriptome for X. laevis. By BLASTing against available amphibian transcriptomes (30-

271 33), we further identified putative Md-2 proteins in Rhinella marina, Hynobius retardatus,

272 Odorrana margaretae, and Ichthyophis bannanicus (Fig S1).

273 With these putative amphibian Md-2 sequences in hand, we returned to our search for a

274 zebrafish Md-2. A BLAST against a zebrafish transcriptome using the $X$. laevis sequence

275 revealed a likely transcript (si:dkey-82k12.13,23\% identity). We then searched additional fish

276 transcriptomes available from the Fish-T1K project (49) and identified a set of transcripts from

277 three species that matched Md-2 (Fig S1). The genes we identified in bony fishes that encode

278 putative Md-2 proteins were highly diverged. On average, they exhibited only $26 \%$ identity

279 against human $\mathrm{Md}-2$, and only $\sim 40 \%$ identity relative to one another.

We next set out to assign the orthology of these putative Md-2 sequences. Our primary

281 concern was that these newly identified sequences were paralogs of Md-2. We therefore built a

282 phylogenetic tree to elucidate whether these newly identified sequences grouped with Md-2 or

283 with its direct paralog, Md-1. We constructed an alignment of 294 Md-1 and Md-2 protein

284 sequences sampled from amniotes, amphibians, and bony fishes and then used this to infer a

285 maximum likelihood phylogeny (Fig 2A). The alignment is available in File S1.

The putative amphibian and bony fish Md-2 sequences grouped with the tetrapod MD-2 sequences with strong support $(\mathrm{SH}=0.99)$. The $\mathrm{Md}-1 / \mathrm{Md}-2$ protein tree largely reproduced the

288 species tree, with the exception of amphibians. On the Md-1 lineage, amphibians form a

289 polytomy with fishes at the base of the tree; on the Md-2 lineage, they are placed inside the 
291 number of amphibian sequences, as well as the rapid evolution of the genes along these lineages.

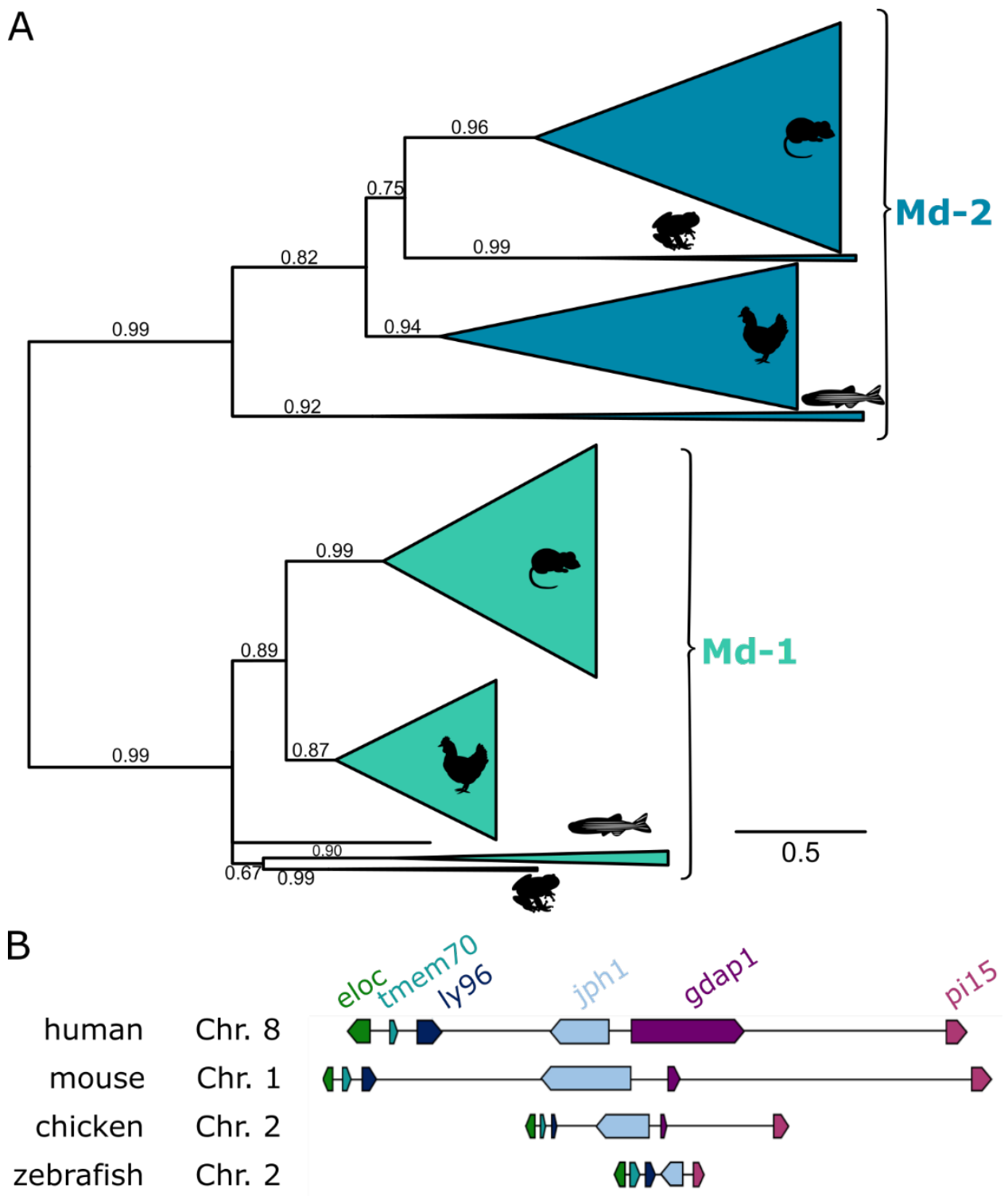

Fig 2. Phylogeny and synteny of the identified zebrafish protein support classifying it as an Md-2 (the ly96 gene). A) Maximum likelihood phylogeny of Md-2 and Md-1 proteins. Wedges are collapsed clades of orthologs, with wedge height corresponding to the number of included taxa and wedge length indicating the longest branch length with the clade. Support values are SH-supports calculated using an approximate likelihood ratio test. Clades are colored to highlight Md-2 (blue) vs. Md-1 (green) classification. The taxa included in each clade are noted on the tree by silhouettes of mammals (mouse), sauropsids (chicken), amphibians (frog), and fish (zebrafish). B) Genomic organization of genes surrounding Md-2 in vertebrates. Arrows for genes represent the coding strand. Approximate distances between genes are represented by the length of line for the selected chromosome. 
Overall, the tree is consistent with a single gene duplication event sometime before the evolution of bony vertebrates. Then, Md-1 and Md-2 were preserved along most descendant

294 lineages. This said, the protein sequences of Md-1 and, particularly, Md-2 are evolving rapidly.

295 The total branch lengths between the last common ancestor of Md-2 to its human and zebrafish

296 descendants are 2.00 and 2.44, respectively. Put another way, the average site in the Md-2

297 sequence has changed its amino acid $\sim 2$ times over the last 430 million years. Only 7 of 160

298 positions in MD-2 are universally conserved across the clade.

To cross-validate the orthology of this newly identified gene, we next examined its

300 locations in the $X$. laevis and $D$. rerio genomes. We found that the synteny is consistent with

301 other bony vertebrates (Fig 2B). In five genomes sampled from across bony vertebrates-

302 including $X$. laevis and D. rerio - the gene encoding Md-2 is located between tmem70 and jph1b

303 (Table S1). This provides strong evidence that these amphibian and fish genes are, in fact,

304 orthologous to the amniote gene encoding Md-2. By convention, the gene encoding Md-2 is

305 known as $l y 96$. We therefore refer to this gene as zebrafish $l y 96$ hereafter.

306 Due to the genome duplication event that occurred along the zebrafish lineage (50), we

307 also looked for a second copy of ly96. We examined the genomic location of the jphla paralog,

308 but we were unable to identify an additional gene with any similarity to ly96. It appears that an

309 inversion may have occurred in this region, complicating identification by synteny alone. This

310 said, no additional transcripts were identified within the zebrafish transcriptome with similarity

311 to the identified zebrafish $l y 96$ sequence. This is consistent with a loss of the duplicate copy of

312 this gene.

313 Finally, we attempted to identify an Md-2 sequence from earlier-diverging lineages

314 including Chondrichthyes (cartilaginous fishes) and Agnatha (jawless fishes). Despite extensive 
315 BLASTing, we were unable to identify an Md-2 protein sequence or $l y 96$ gene in either lineage.

316 This is consistent with $l y 96$ arising after the divergence of cartilaginous and bony fishes ( 470

317 million years ago), but before the divergence of bony- and ray-finned fishes ( $\sim 35$ million years

318 ago). The sequence resources for cartilaginous and jawless fishes remain relatively sparse,

319 however, so we cannot exclude an earlier origin for $l y 96$.

321 Zebrafish transcribe $l y 96$ in innate immune cells

322 We next asked whether zebrafish express $l y 96$. To do so, we used the recently released

323 Zebrafish Single-Cell Transcriptome Atlas (44). This dataset consists of single-cell RNASeq

324 transcriptomes for 44,102 individual cells extracted from 1, 2 and 5 dpf zebrafish. The gray

325 points in Fig 3A and 3B shows the entire Atlas: each point is a cell, plotted such that cells with

326 similar transcription profiles appear near one another. Cluster identity can be established by

327 examining differentially expressed transcripts and using these marker genes to assess cell type

328 expression in vivo (44); this provides a means to assess which cell types express $l y 96$ simply by

329 asking which clusters possess $l y 96$ transcripts. 
A

C

A

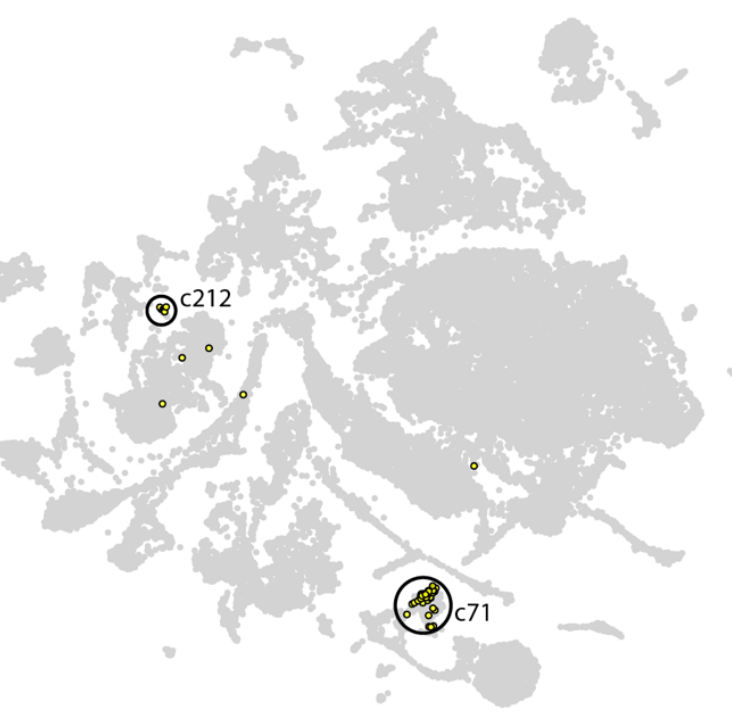

B

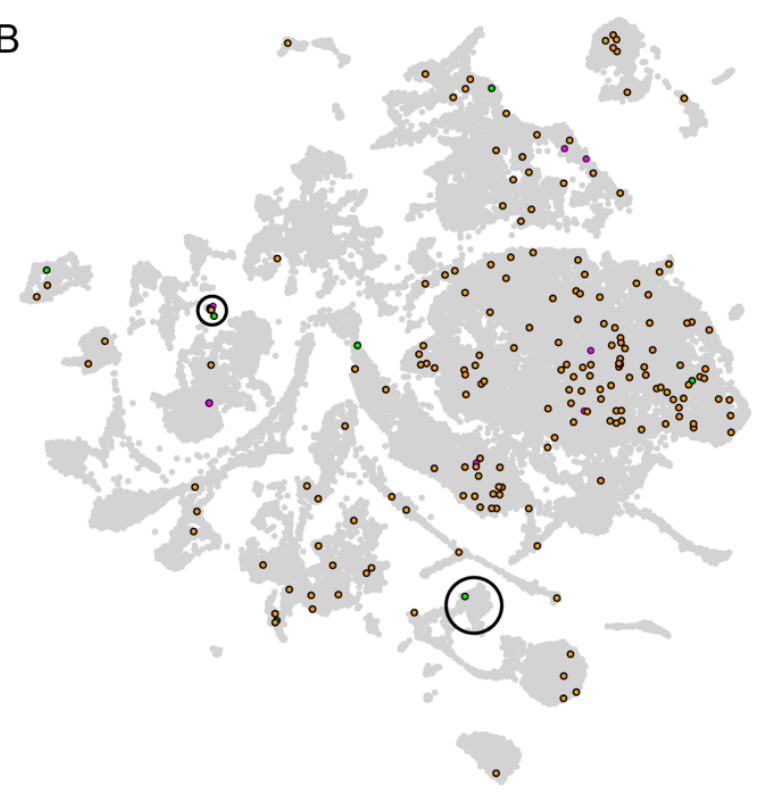

Fig 3. $l y 96$ and $t$ lr 4 genes are expressed in macrophage cells. Each point in these plots is an individual cell characterized by single-cell RNA-Seq. The distance between the cells corresponds to the relative difference in their transcriptional profiles (44). A) Yellow points indicate cells expressing $1 y 96$, gray points show all 44,102 cells in the data set. The two clusters in which $l y 96$ is expressed (c71 and c212) are highlighted with black circles. B) Colored points indicate cells expressing $t l r 4 b b$ (green), tlr4al (magenta), or tlr4ba (orange); gray points and circles are identical to panel A. C) Enlarged views of clusters c212 and c71, separated by gene of interest. This includes the genes shown in panels $\mathrm{A}$ and $\mathrm{B}$, as well the macrophage marker, mpeg1.1 (light blue) $(26,51)$. The number in the bottom left of each table entry is the expression level of the gene within the cluster divided by its expression level in all other cells in the dataset. If there was no expression in cells outside the cluster, expression within the cluster was divided by the detection threshold (0.001) giving a minimum estimate for the enrichment. The background cells are now colored by the developmental stage from which the cell was isolated: $1 \mathrm{dpf}$ (white), 2 dpf (light gray), or $5 \mathrm{dpf}$ (dark gray). The dashed lines in shown on c71 are approximate divisions between the age-dependent sub-clusters of $\mathrm{c} 71$. yellow points). Both of these clusters are annotated in the Atlas as putative macrophage cells 
350 based on their transcription profiles (44). ly96 is highly enriched in these clusters relative to other

351 clusters. This can be measured by taking the ratio of the average expression level of $l y 96$ for the

352 cells in the cluster relative to the average expression level of $l y 96$ in all other cells. This ratio is

353235 for cluster $\mathrm{c} 212$ and $\geq 173$ for cluster $\mathrm{c} 71$, indicating that $l y 96$ is highly enriched in these

354 clusters. For comparison, the well-established macrophage marker mpeg1.1 $(26,51)$ has ratios of

355102 and 202, respectively, for these same clusters (Fig 3C).

356 We next investigated the expression of the tlr4bb, tlr4al, and tlr4ba genes. We found that

$357 t l r 4 b b$ and $t l r 4 a l$ had quite limited expression patterns (Fig 3B, green and pink), while tlr4ba was

358 expressed broadly (Fig 3B, orange). All three tlr4 genes were found in cluster c212, but only

$359 t$ tr4bb was found in cluster c71 (Fig 3C).

The Atlas also has the potential to reveal time-course information for the expression of

361 these genes, as it contains cells isolated from fish at 1, 2 and $5 \mathrm{dpf}$. We therefore shaded the cells

362 within clusters c71 and c212 by their developmental time point (Fig 3C). Cluster c212, where we

363 observed overlapping expression for $l y 96$ and all three $t l r 4$ genes, consists entirely of cells

364 isolated from $5 \mathrm{dpf}$ zebrafish (Fig 3C). Cluster c71 has three discrete sub-clusters corresponding

365 to the age of the fish from which the cell was extracted. We see no ly96 in the $1 \mathrm{dpf}$ sub-cluster,

366 a small amount in the $2 \mathrm{dpf}$ sub-cluster, and the highest level in the $5 \mathrm{dpf}$ sub-cluster (Fig 3C).

367 Likewise, tlr4bb is expressed in the 5 dpf sub-cluster but no others. For comparison, the

368 macrophage marker mpeg1.1 is found in all cells within c71 and c212, regardless of the age of

369 the fish from which the cell was extracted.

370 These observations suggest that $l y 96$ and all three tlr 4 genes are expressed together in a

371 subset of macrophage cells by $5 \mathrm{dpf}$ (Fig 3C, c212). Samples of later time points would be 
372

373

374

375

376

377

378

379

380

381

382

383

384

385

386

387

388

389

390

391

392

393

394

necessary to establish if these genes are at their full expression level by $5 \mathrm{dpf}$, or if their expression level and cell-type specificity continues to change as the fish develop.

\section{Zebrafish Tlr4a/Md-2 can activate NF- $\mathrm{kB}$ in response to lipopolysaccharide}

Given the low sequence similarity between the zebrafish Md-2 protein and its amniote orthologs, it was not clear that the zebrafish Md-2 would be capable of mediating the Tlr4 response to Md-2. We therefore turned to an ex vivo cell culture assay to assess the ability of the zebrafish Md-2 to partner with zebrafish Tlr4a and Tlr4b for LPS activation. In this assay, we cotransfected genes encoding complex components into HEK293T cells and then used luciferase to

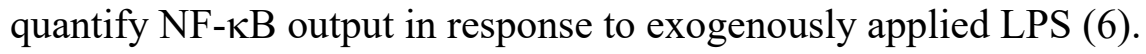

We started by co-transfecting cells with zebrafish $l y 96$ and zebrafish tlr $4 b a$ or $t l r 4 b b$ and then measuring NF- $\mathrm{B}$ activation in response to exogenously applied LPS. We saw no activation (Fig 4A). This result was unsurprising, as this experiment attempted to activate a Tlr4/Md-2 complex without Cd14 - an important peripheral protein that brings LPS to Tlr4/Md-2 complexes in amniotes, dramatically increasing the NF- $\mathrm{KB}$ response (Fig 1) (52-56). We thus co-transfected $t l r 4 b a$ or $t l r 4 b b$ with zebrafish $l y 96$ and human $c d 14$. In this context, we observed potent activation of NF- $\kappa \mathrm{B}$ in response to LPS for $t$ lr $4 b a$, but not $t \operatorname{lr} 4 b b$ (Fig 4A). To verify that the activation of Tlr4a required Md-2, rather than merely Cd14, we tested the activation of Tlr4 and Cd14 without transfecting ly96 - this complex did not respond to LPS (Fig 4A). We then verified that the zebrafish Tlr4a/Md-2 complex, complemented with human Cd14, exhibited a dose-dependent response to LPS (Fig 4B). The concentration of LPS needed for activation of the zebrafish Tlr4a/Md-2 complex was much higher than that needed for activation of the human proteins in these cells, but consistent with what has been observed for other species (57). 

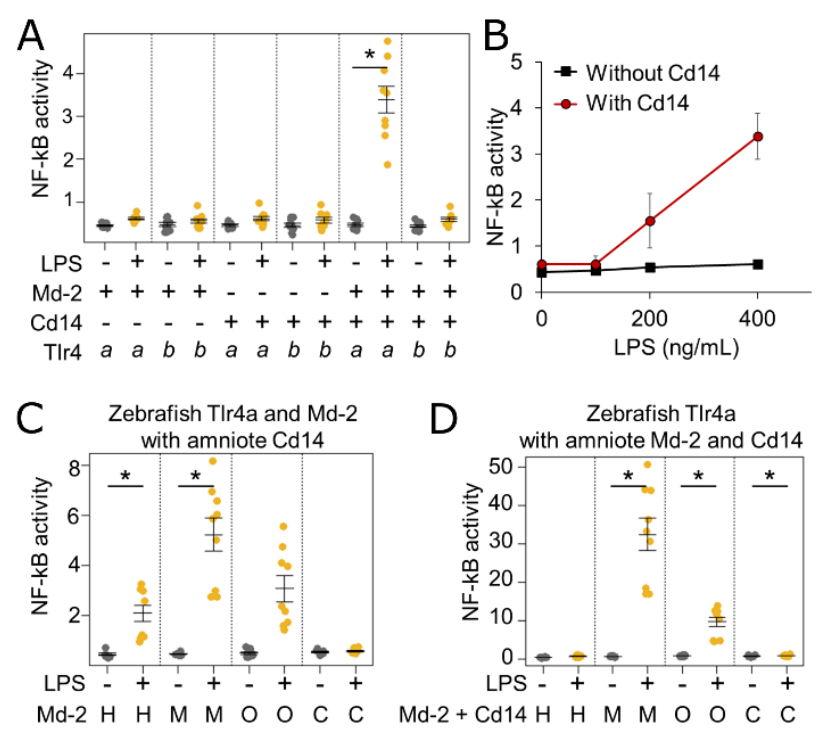

Fig 4. LPS activates the zebrafish Tlr4a/Md-2 in a functional assay. A) Activation of zebrafish Tlr4a and Tlr4b in the presence and absence of zebrafish Md-2 and human CD14. Points are the technical replicates from three biological replicates. Bold lines are the mean of the biological replicates. Error bars are a standard error on the mean of the biological replicates. B) Dose-dependence of LPS response by zebrafish Tlr4a/Md-2 in the presence (red) and absence (black) of human CD14. C) Zebrafish Tlr4a/Md-2 complemented with Cd14 proteins from amniotes. D) Zebrafish Tlr4a complemented with species-matched Md-2/Cd14 pairs taken from amniotes. Statistically significant differences (single-tailed Student's t-test) are noted on each panel $(* \mathrm{p}<0.05)$

Our results support the hypothesis that zebrafish $\mathrm{Tl} 4 \mathrm{a} / \mathrm{Md}-2$ can activate in response to LPS; however, this could only be done with the presence of a supporting mammalian protein (human Cd14). To determine if this was an artifact of the human protein, we tested the LPS activation of Tlr4a/Md-2 in the presence of human, mouse, opossum, and chicken Cd14. We found that all but the chicken Cd14 were able to support the activation of the complex (Fig 4C). Thus, the activity of the zebrafish Tlr4a/Md-2 complex does not depend exclusively on human Cd14 but can instead be supported by diverse Cd14 molecules. Given the importance of Cd14 in this assay, we looked for evidence of a zebrafish $c d 14$ gene; however, we were unable to locate such a gene. The inability to detect a $c d 14$ in fish may be due to rapid evolution of this gene since the most recent common ancestor, or, alternatively, Cd14 may have arisen as a supporting 
417 molecule for LPS-recognition after the divergence of tetrapods. The requirement for Cd14 in

418 these experiments could be a problem with the heterologous cell line (these experiments were

419 done in human cells) or a missing alternate secondary cofactor (such as a fish LPS binding

420 protein).

421 Finally, to see if zebrafish Tlr4a behaved similarly to amniote Tlr4, we investigated

422 whether Md-2 from other species could act in concert with zebrafish Tlr4a. We co-transfected

423 tr $4 a$ with human, mouse, or opossum $1 y 96$ genes. We saw complementation by both mouse and

424 opossum Md-2 for LPS activation of zebrafish Tlr4a (Fig 4D). This suggests that the

425 requirements for activation by the Tlr4/Md-2 complex have been conserved for over 400 million

426 years and are shared across bony vertebrates.

427

\section{Md-2 is not required for LPS-induced death in 5 dpf larval zebrafish}

We next probed the physiological role of Md-2 in LPS-induced septic shock in larval 5

430 dpf zebrafish. We first treated 5 dpf larval WT zebrafish with LPS and followed their survival

431 over time. No treated WT fish survived more than 48 hours; the median survival time was 30 hrs

432 (Fig 5A). As a control, we also tested the LPS response for $m y d 88^{-/-}$fish. As has been observed

433 previously (22), these showed a modest but significant increase in survival (Fig 5A). This was

434 consistent with LPS inducing a response that depends in part on a myd88 dependent pathway.

435 To test the role of Md-2 in this response, we used CRISPR-Cas9-based mutagenesis to

436 establish three independent zebrafish lines with mutations in the first exon of the $l y 96$ gene. The

437 mutations were expected to induce a loss of function through removal of the start codon $\left(\operatorname{ly} 96^{A / A}\right)$

438 or through a frame shift and premature stop codon $\left(l y 96^{B / B}\right.$ and $\left.l y 96^{C / C}\right)$ (Table S2). Using RT-

439 PCR primers downstream of the targeted region, we demonstrated that $l y 96 \mathrm{mRNA}$ is expressed

440 in mutant larval zebrafish (Fig 5E). 
We then tested the three $l y 96$ mutant zebrafish lines for their susceptibility to LPS-

443 zebrafish survived for slightly longer (Fig 5B); ly96 $6^{B / B}$ zebrafish survived similarly (Fig 5C), and

$444 \quad l y 96^{C / C}$ zebrafish survived shorter (Fig 5D). This is consistent with some other pathway rather

445 than Tlr4/Md-2 being the primary route for LPS-induced death in larval zebrafish.

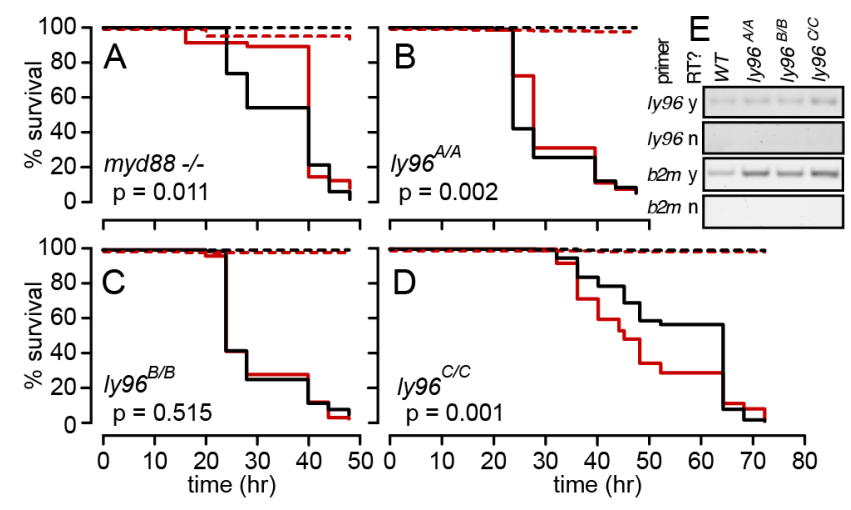

Fig 5. ly96 mutations only moderately affect LPS survival in larval zebrafish. A-D) Curves show survival of wildtype (black) and mutant (red) zebrafish in the presence of $0.6 \mathrm{mg} / \mathrm{mL}$ LPS (solid line) or mock treatment (dashed line). The genotype is indicated on each panel. The pvalue was determined by comparing the matched survival curves by a log-rank Mantel-Cox test. The experiments shown in panels A-C were performed in parallel, while the experiments in panel D were performed at a later date with an LPS lot that showed lower potency, necessitating a longer treatment time. Panels A-D represent averages of one, five, five, and three experimental repeats, respectively. Panel E shows mRNA transcript level for each mutant zebrafish. Rows use different primers (ly96 or $b 2 \mathrm{~m}$ ) with and without reverse transcriptase (RT). Columns show fish genotype.

\section{The zebrafish $t$ lr4 paralog arose after the evolution of $l y 96$}

Finally, we revisited the idea that the evolutionary history of zebrafish $t$ tr 4 genes implies that they do not act as LPS-sensing molecules. Previous authors suggested that an ancestral TLR gene duplicated in the ancestor of bony vertebrates ( $\sim 450$ million years ago), and that the two paralogs were then differentially lost on the mammalian and bony fish lineages (21), respectively. This early divergence, before the evolution of $l y 96$, may suggest very different functional roles for mammalian versus fish $t$ lr $4 \mathrm{~s}$. 
We set out to better resolve when the zebrafish tlr4 paralog arose relative to its

mammalian counterparts, particularly with regard to the evolution of $l y 96$. As with our analysis

468 of Md-2, we started with a phylogenetic tree and then turned to synteny. For the phylogenetic

469 tree, we constructed a multiple sequence alignment containing 263 Tlr4 sequences and 190

$470 \mathrm{Cd} 180$ protein sequences as an outgroup. (Cd180 is the most closely related paralog to Tlr4)

471 (58). The alignment is available in File S2. In the resulting maximum likelihood tree, Tlr4 and

472 Cd180 form distinct, well-supported clades (Fig 6A). Within the Tlr4 clade, zebrafish Tlr4a and

473 Tlr4b are part of a monophyletic group with other Tlr4s.

We next investigated the genomic context for tlr4 genes in eleven genomes, each with a complete chromosome assembly (Table S3). We selected a set of 22 genes flanking human $t$ lr4

476 and a set of 22 genes flanking the three zebrafish tlr4 genes (Table S4). Notably, there were no

477 shared homologs between the sets, demonstrating the radical difference between the genomic

478 contexts of human and zebrafish $t$ tr 4 . We then used these sets of genes to BLAST each of the

479 eleven genomes and calculated a running average for the BLAST e-values along each

480 chromosome. This allowed us to assess the overall similarity of genomic regions to either the

481 human or zebrafish tlr4 context. Fig 6B-G shows representative traces for six chromosomes

482 taken from five species, with results for all genomes in Fig S2-S13. We were able to distinguish

483 two distinct contexts for tlr4 genes. In some organisms - human and frog, for example - tlr4 is

484 surrounded by hits from the human gene set (Fig 6B and D). In other organisms_-zebrafish and

485 pike, for example - tlr4 is surrounded by hits from the zebrafish gene set (Fig 6C and E).

To place our results in their evolutionary context, we plotted our BLAST output against

487 the phylogeny for our chosen species. For each species, we displayed the chromosome with the

488 most hits from the human set (Fig 6H) and the chromosome with the most hits from the zebrafish 
set (Fig 6I). We made an exception for the pike, displaying the chromosome with the tlr4 gene

490 (linkage group 5), not the chromosome with the most zebrafish hits (linkage group 6). We

491 indicated whether a gene from the human or zebrafish set was seen somewhere on that

492 chromosome by coloring the square corresponding to that gene.

494 these species - including the gar-had a duplicate copy of tlr4 in a zebrafish-like context. The

495 human-like context of the gar gene is shown Fig 6F, while the lack of tlr4 in the most zebrafish-

496 like region of the gar genome is shown in Fig 6G. The remainder of the ray-finned fishes had tlr4

497 in either a zebrafish-like context (catfish, zebrafish, and pike) or had no tlr4 gene at all

498 (bonytongue, cod, and puffer).

The most parsimonious history consistent with the observed distribution across genomes

500 is shown in Fig 6J. In this scenario, tlr4 arose in a genomic context similar to the one preserved

501 in humans. This occurred after the divergence of bony and cartilaginous fishes ( 475 million

502 years ago), but before the divergence of ray-finned and lobe-finned fishes ( $\sim 30$ million years

503 ago). The ancestral genomic context was preserved in tetrapods, including humans. It was also

504 maintained in the ray-finned fishes for $\sim 130$ million years, as indicated by the location of the $t \operatorname{tr} 4$

505 gene in the gar genome. Then, sometime between 300 and 250 million years ago, the tlr4 gene

506 was both duplicated into the genomic context observed in zebrafish, as well as lost from the

507 ancestral context. Finally, between 150 and 100 million years ago, a tandem duplication occurred

508 within the Cypriniformes fishes, leading to the tandem copies of tlr4 observed in zebrafish, carp,

509 and other Cypriniformes fishes. 


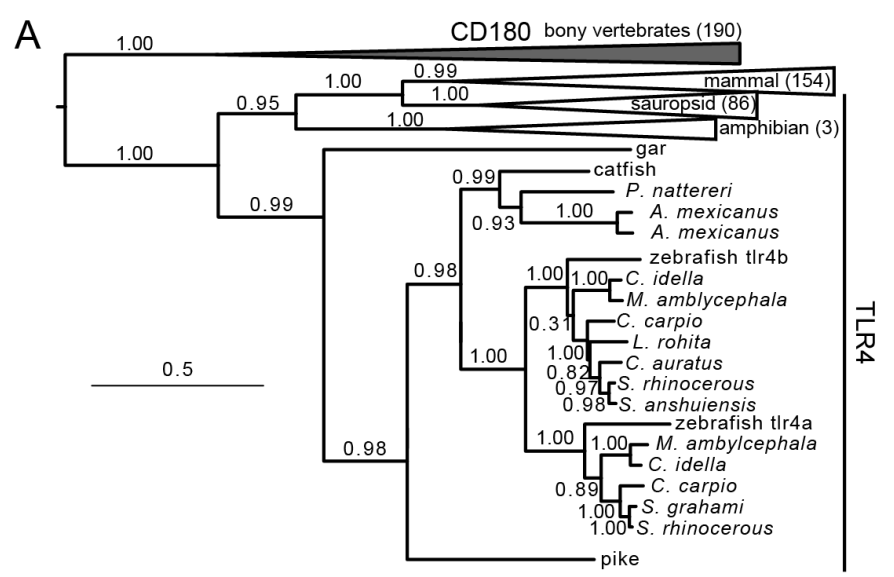

$\mathrm{H}$

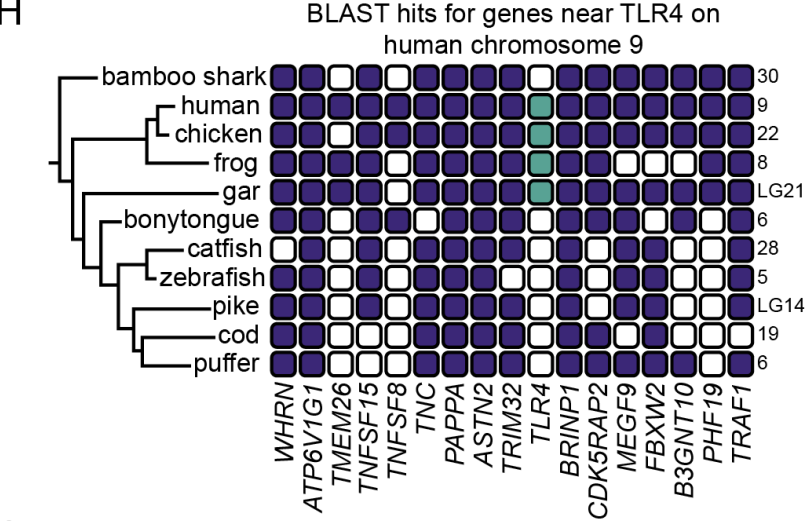

I

BLAST hits for genes near TLR4 on zebrafish chromosome 13

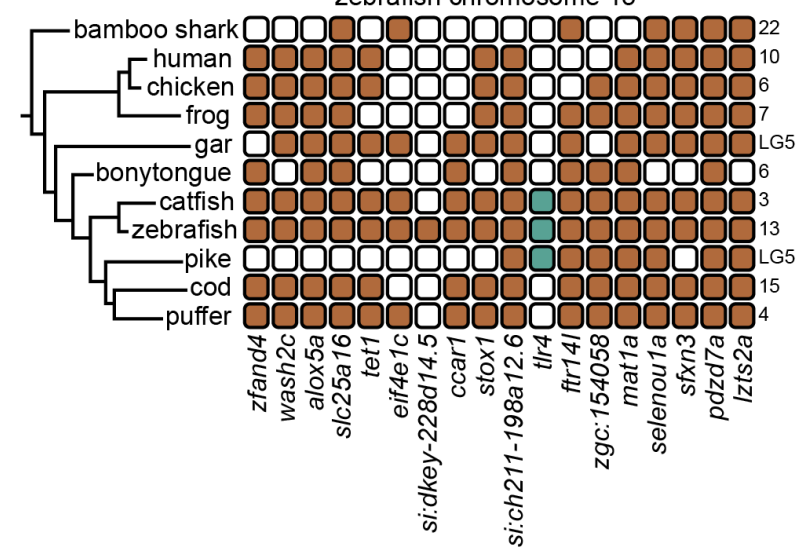

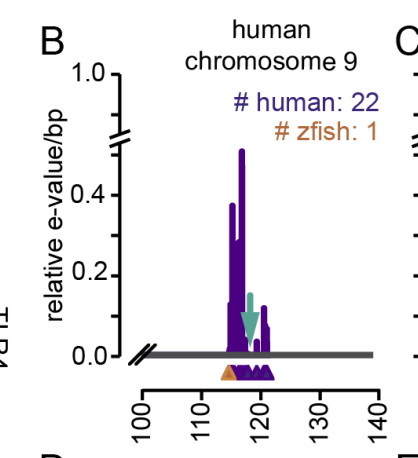
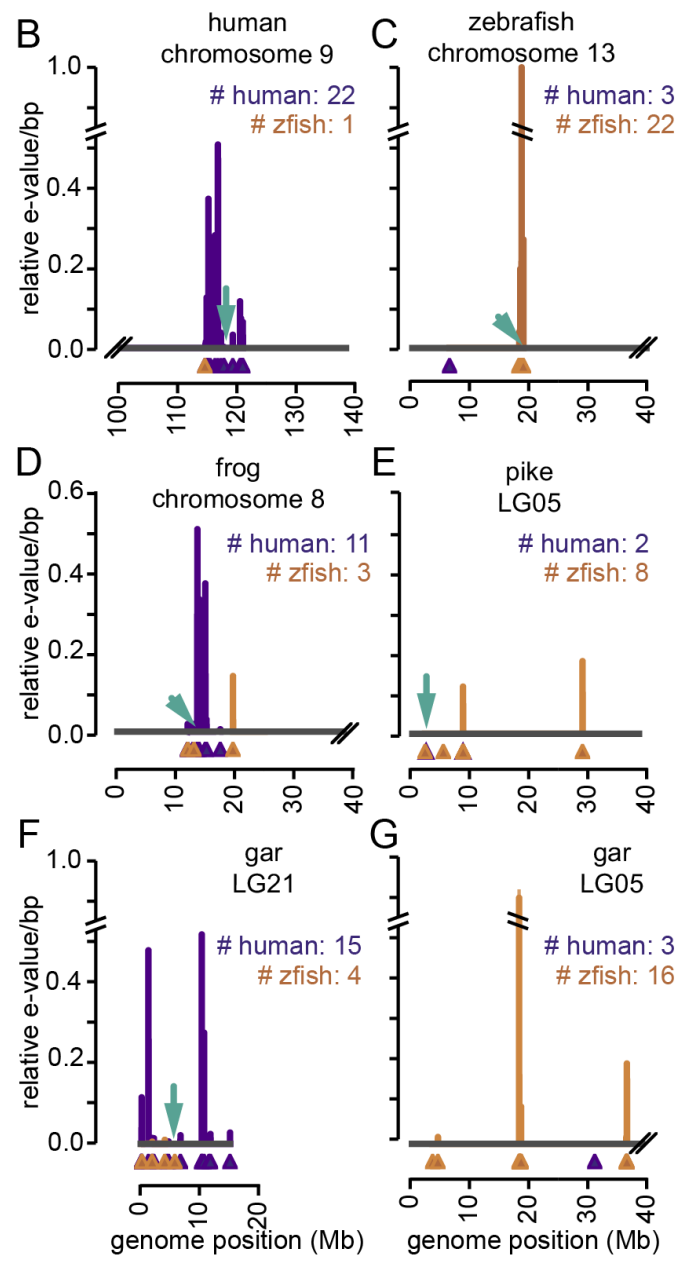

E

1 pike
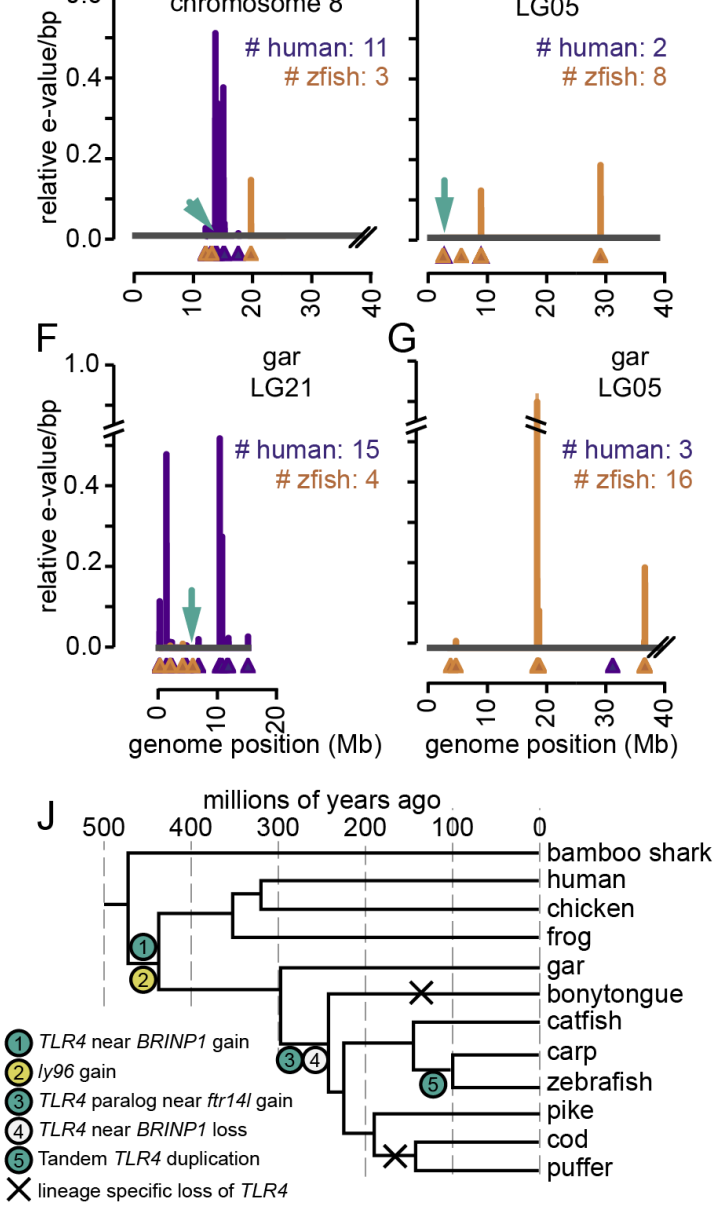

Fig 6. Zebrafish tlr4 paralogs evolved within the ray-finned fishes. A) Maximum likelihood phylogeny for $453 \mathrm{Tlr} 4$ and Cd180 protein sequences. SH supports are indicated on the tree. Wedges are clades, with the length indicating the maximum branch length from the ancestor of the clade. The taxonomic distribution and number of genes within each wedge are indicated on the plot. B-G) Hits for human (purple) and zebrafish (orange) gene sets on six representative chromosomes taken from five species. The species and chromosome are indicated at the top of each plot. The $\mathrm{x}$-axis denotes position on the chromosome. Triangles indicate gene start positions. The green arrow indicates the location of a Tlr4 gene. The y-axis is a running average of the BLAST e-value for each gene set along the genome (see methods). The numbers on the plot indicate the number of human and zebrafish hits within the region shown. H,I) Each row 
shows the chromosome with the most BLAST hits from the human (panel H) or zebrafish (panel I) gene set. Columns indicate specific genes from the set, with names denoted below. A colored square indicates a gene found somewhere on the chromosome. A green square is a tlr4 gene. The species tree is shown on the left; the chromosome number is on the right. J) Schematic representation of a plausible scenario for the history of the $t l r 4$ gene. Times are taken from Hughes et al. (59) and timetree.org (60).

This revised evolutionary history places the evolution of the zebrafish tlr4 paralogs much later than was previously hypothesized (21). Importantly, the duplication of TLR4 occurred after the evolution of Md-2, meaning that the formation of the Tlr4/Md-2 complex likely pre-dates the duplication event. Thus, the interaction with Md-2 and the ability to activate with LPS were an ancestral feature of zebrafish Tlr4 rather than something that could only be gained in parallel along the tetrapod and bony fish lineages.

\section{DISCUSSION}

Our observations led us to reevaluate the decade-old idea that Tlr4 does not participate in the LPS-induced inflammatory response in zebrafish. We have identified the zebrafish gene encoding the Tlr4 co-receptor Md-2 (ly96). The gene, like tlr4ba and tlr4bb, is transcribed in zebrafish cells that transcribe a collection of macrophage genes. In concert with zebrafish Tlr4a, zebrafish Md-2 is capable of activating NF- $\mathrm{BB}$ signaling in an ex vivo functional assay. Finally, a careful phylogenetic analysis suggests that the mammalian and zebrafish $t$ tr 4 genes are not as evolutionarily distinct as previously thought. While not direct orthologs, the zebrafish paralogs evolved well after $l y 96$ and likely preserve an ancestral LPS recognition activity.

Our work demonstrates that, given the correct context, zebrafish Tlr4a and Md-2 form a functional complex that recognizes LPS and activates NF- $\mathrm{B}$ signaling. Further, the molecular basis for the interaction between the partners appears to have been conserved for the last 450 million years - zebrafish Tlr4a is compatible with mouse and opossum Md-2 (Fig 4D). This is 
550 despite the fact that the orthologous proteins from each species have only $\sim 20 \%$ identity at the

551 amino acid sequence level. The simplest explanation for this observation is that the ability of

$552 \mathrm{Tl} 4 / \mathrm{Md}-2$ to activate in response to LPS is an ancestral feature of the protein complex that has

553 been conserved across the bony vertebrates - from mammals to bony fishes.

554 We have not shown, however, that LPS-induced Tlr4/Md-2 signaling actually occurs in

555 zebrafish. Our two attempts to do so- our cell culture functional assay and analysis of zebrafish

556 ly96 loss of function mutants - both gave mixed results. We will discuss each in turn.

\section{LPS activation of Tlr4a/Md-2 in human cells requires supporting molecules}

In our functional assays, we had to add a mammalian Cd14 to activate NF- $\kappa \mathrm{B}$ signaling

561 We could find no ortholog to $c d 14$ in the zebrafish genome.

One possibility is that the human cell line used for the functional assays is missing some

563 critical component for the delivery of LPS and assembly of the active dimer. Tlr4, Md-2, and

564 Cd14 are the necessary and sufficient set of amniote proteins that confer an LPS-dependent NF-

$565 \kappa \mathrm{B}$ response in HEK293T cells. It could be that some other non-homologous protein plays the

566 role of Cd14 in zebrafish.

Another possibility is that LPS is not a zebrafish Tlr4a/Md-2 agonist in vivo. We showed

568 that we can activate the complex in a human cell line given an appropriate delivery molecule and

569 a high enough LPS concentration. But, under physiological conditions, the Tlr4a/Md-2 complex

570 could respond to some other chemically similar ligand. This would not be surprising: changes in

571 ligand specificity have been observed across Md-2 in the amniotes (61). There is also some

572 evidence that zebrafish Tlr4a may be antagonized by LPS in vivo (20). This would be compatible 
573 with another ligand activating the complex and LPS competing and activating at a lower level

574 than can be achieved by the native ligand.

575 Finally, our observation that Tlr4a activates NF- $\kappa$ B with both mouse and opossum Md-2

576 directly contrasts previous work that showed the complex could not activate (Fig 4C) (20, 21).

577 The key difference between our experiments and those done previously is the sequence of $t \operatorname{tr} 4 a$

578 used. Previous investigators used a construct that was $\sim 75$ amino acids shorter than tetrapod

579 Tlr4s. This construct is missing both the signal peptide required to target Tlr4a to the cell surface

580 and a region of the protein that is likely critical for Md-2 binding (Fig S13). In contrast, we used

581 a full-length ORF (ENSDART00000044697.6, GRCz10). The difference in our constructs arises

582 because the previous analysis relied on cDNA that, apparently, captured an alternate splice

583 variant of $t l r 4 a$.

Multiple pathways contribute to LPS-induced death in larval zebrafish

Larval zebrafish ly96 loss of function mutants did not exhibit appreciably altered death

587 rates upon exposure to LPS compared to WT (Fig 5B-D). This is consistent with a previous

588 morpholino study that knocked down tlr4a and observed no change in sensitivity to LPS (20).

589 This contrasts with mice, however, where knockout of $L Y 96$ is protective against endotoxic

590 shock (4) and disruption or knockout of Tlr4 leads to hypo-responsiveness to LPS (1, 62).

We cannot rule out the possibility that this lack of response is due to an experimental

592 artifact. First, zebrafish may have retained a second copy of the $l y 96$ gene from the teleost

593 genome duplication that maintained function even after deletion of the targeted copy. We were

594 unable find any evidence of such a gene; however, the challenge of finding the original ly96 gene

595 means that we cannot rule this out. A second possibility is that the mutants that we generated 
may not represent a complete loss of function. For example, use of a potential alternative start codon 17 amino acids downstream of the normal start codon could produce a truncated protein

$598\left(l y 96^{A / A}\right)$. Although this would be missing N-terminal amino acids that are known to be critical

599 for Md-2 function in other systems (Table S2), these amino acids may not be necessary in

600 zebrafish. Finally, we tested a single developmental time point. It could be that Tlr4a/Md-2,

601 while expressed in larvae (Fig 3C), is not yet a large player in LPS sensing. Further experiments

602 on zebrafish at different time points may help clarify this point.

Another challenge is that LPS-mediated death is a relatively blunt instrument to test for

604 the activity of the Tlr4/Md-2 complex. We observed that addition of LPS dramatically increased

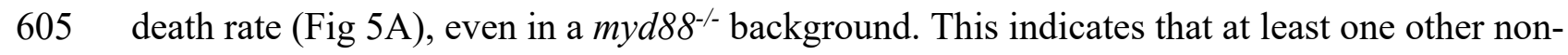

606 Toll-like pathway contributes to LPS-induced. One possibility is that this occurs by intracellular

607 sensing of LPS via caspases and inflammasomes (63). Various studies have shown

608 inflammasome signaling to be widespread in zebrafish larvae (64) and Il-1r to be required to

609 prevent cell death in response to infection in multiple cells (65). Intracellular sensing may be

610 much more important in fish than mammals: zebrafish have 385 of these putative intracellular

611 sensors whereas humans have $22(66)$.

As a result of such alternate pathways, even if the Tlr4a/Md-2 complex contributes to the

613 LPS-induced inflammatory response, removing it might not lead to a measurable difference in

614 death rate. Compounding this difficulty, our expression analysis revealed that zebrafish $l y 96$ is

615 much more restricted in its expression than the corresponding mammalian genes (27). There

616 may, in fact, be specific subtypes of macrophages that express $l y 96$ and $t l r 4 s$ - and are defective

617 in LPS sensing in the ly96 mutants_-but remain invisible at the level of LPS-induced death.

618 Higher-resolution studies of LPS-induced inflammation will be required to sort this out. 


\section{Snapshot in the evolutionary history of this complex}

The presence of Md-2 in zebrafish indicates that both Tlr4 and Md-2 existed, together, in

622 the last common ancestor of bony vertebrates. Because descendants along both the tetrapod and

623 ray-finned fish lineages activate with LPS, the ability to respond to LPS is likely an ancestral

624 function that has been conserved for 435 million years.

That said, these proteins have evolved significantly since this shared ancestor. Along the tetrapod lineage, a supporting collection of proteins evolved. Cd14 arose through a duplication within the Toll-like receptor family and is now an essential component of the Tlr4/Md-2 complex, delivering LPS to Md-2 in a coordinated fashion $(53,54)$. Tetrapods also acquired Lipid Binding Protein (LBP), improving LPS delivery $(52,67)$. Amniotes then further adjusted the Tlr4/Md-2 pro-inflammatory response through addition of amniote-specific Damage-

631 Associated Molecular Pattern (DAMP) molecules such as S100A9 (68), which amplify LPS-

632 induced inflammation (69). All the while, mutations to Md-2 changed its specificity for LPS and 633 its chemical analogs (70). For example, humans acquired unique lipid IVa antagonism sometime 634 after the divergence of humans and mice $(71,72)$.

636 acquire supporting LPS delivery molecules analogous to Cd14? Has the specificity of Md-2

637 fluctuated in ray-finned fishes as it has along the tetrapod lineage? Further work is needed to 638 answer these questions. 
642 75-77). This parallels the observation that early diverging tetrapods, such as amphibians, also

643 require high doses of LPS to trigger an inflammatory response (78). This could be explained if

644 ray-finned fishes do not have specialized machinery to deliver LPS to the complex, but instead

645 use Tlr4/Md-2 as a simple LPS sensor. Other observations consistent with a relatively primitive

646 Tlr4/Md-2 LPS response in zebrafish are the fact that Tlr4 was lost, independently, along

647 multiple fish lineages $(20,21,73)$ (Fig 6J), as well as the existence of parallel LPS sensing

648 pathways in zebrafish (64). If the Tlr4/Md-2 complex is peripheral to the LPS response in ray-

649 finned fishes, it could be lost with minimal fitness consequences. In contrast, Tlr4/Md-2 became

650 progressively more central to the LPS response along the mammalian lineage - and as a result

651 has been highly conserved.

652 Our work suggests that we should re-visit our understanding of LPS signaling through

653 Tlr4/Md-2 in zebrafish. We hypothesize that zebrafish preserve an ancestral, low-sensitivity

654 Tlr4/Md-2 complex. In contrast to mammals - in which the Tlr4/Md-2 complex is the primary

655 LPS receptor - the zebrafish Tlr4/Md-2 complex acts in parallel with several LPS-sensitive

656 pathways, likely playing roles in a small population of innate immune cells.

\section{ACKNOWLEDGMENTS}

659 We thank Kristi Hamilton and Lila Kaye for assistance with zebrafish LPS survival assays, and

660 Rose Sockol and the University of Oregon Aquatic Animal Care Services staff for fish

661 husbandry. We thank Prof. Carol Kim for sharing the tlr4bb plasmid.

\section{REFERENCES}



in Tlr4 Gene. Science (80-. ). 282: 2085-2088. endotoxin activity. Arch. Microbiol. 164: 383-389. by Bacterial Endotoxin. Annu. Rev. Immunol. 13: 437-457.

672 M. Kimoto, and K. Miyake. 2002. Essential role of MD-2 in LPS responsiveness and TLR4

673 distribution. Nat. Immunol. 3: 667.

674 5. Park, B. S., and J.-O. Lee. 2013. Recognition of lipopolysaccharide pattern by TLR4

675 complexes. Exp. Mol. Med. 45: e66.

676 6. Chow, J. C., D. W. Young, D. T. Golenbock, W. J. Christ, and F. Gusovsky. 1999. Toll-like

677 receptor-4 mediates lipopolysaccharide-induced signal transduction. J. Biol. Chem. 274: 10689-

67892.

679 7. Yiu, J. H. C., B. Dorweiler, and C. W. Woo. 2017. Interaction between gut microbiota and 680 toll-like receptor: from immunity to metabolism. J. Mol. Med. (Berl). 95: 13-20.

681 8. Fan, J., and A. B. Malik. 2003. Toll-like receptor-4 (TLR4) signaling augments chemokine682 induced neutrophil migration by modulating cell surface expression of chemokine receptors. Nat. 683 Med. 9: 315-321.

684 9. Murad, S. 2014. Toll-Like Receptor 4 in Inflammation and Angiogenesis: A Double-Edged 685 Sword. Front. Immunol. 5: 313. 
10. Leon, C. G., R. Tory, J. Jia, O. Sivak, and K. M. Wasan. 2008. Discovery and development of toll-like receptor 4 (TLR4) antagonists: a new paradigm for treating sepsis and other diseases. Pharm. Res. 25: 1751-61.

694 on both Myeloid Cells and Dendritic Cells Is Required for Systemic Inflammation and Organ

695 Damage after Hemorrhagic Shock with Tissue Trauma in Mice. Front. Immunol. 8: 1672.

696 13. Gruffaz, M., K. Vasan, B. Tan, S. Ramos da Silva, and S.-J. Gao. 2017. TLR4-Mediated

697 Inflammation Promotes KSHV-Induced Cellular Transformation and Tumorigenesis by

698 Activating the STAT3 Pathway. Cancer Res. 77: 7094-7108.

699 14. Koliaraki, V., N. Chalkidi, A. Henriques, C. Tzaferis, A. Polykratis, A. Waisman, W. Muller,

700 D. J. Hackam, M. Pasparakis, and G. Kollias. 2019. Innate Sensing through Mesenchymal

701 TLR4/MyD88 Signals Promotes Spontaneous Intestinal Tumorigenesis. Cell Rep. 26: 536-

$702 \quad 545 . e 4$.

703 15. Yoder, J. A., M. E. Nielsen, C. T. Amemiya, and G. W. Litman. 2002. Zebrafish as an

704 immunological model system. Microbes Infect. 4: 1469-1478.

705 16. Renshaw, S. A., and N. S. Trede. 2012. A model 450 million years in the making: zebrafish 706 and vertebrate immunity. Dis. Model. Mech. 5: 38-47. 

zebrafish reporter lines reveal conserved Toll-like receptor signaling potential in embryonic myeloid leukocytes and adult immune cell lineages. J. Leukoc. Biol. 85: 751-765.

711 interactions from proteins to populations. Curr. Opin. Microbiol. 38: 137-141.

712 19. Park, B. S., D. H. Song, H. M. Kim, B.-S. Choi, H. Lee, and J.-O. Lee. 2009. The structural

713 basis of lipopolysaccharide recognition by the TLR4-MD-2 complex. Nature 458: 1191-1195.

714 20. Sepulcre, M. P., F. Alcaraz-Pérez, A. López-Muñoz, F. J. Roca, J. Meseguer, M. L. Cayuela, 715 and V. Mulero. 2009. Evolution of lipopolysaccharide (LPS) recognition and signaling: fish

716 TLR4 does not recognize LPS and negatively regulates NF-kappaB activation. J. Immunol. 182: $717 \quad 1836-45$.

719 and C. H. Kim. 2009. The Gene History of Zebrafish tlr4a and tlr4b Is Predictive of Their

720 Divergent Functions. J. Immunol. 183: 5896-5908.

721 22. Bates, J. M., J. Akerlund, E. Mittge, and K. Guillemin. 2007. Intestinal Alkaline Phosphatase

722 Detoxifies Lipopolysaccharide and Prevents Inflammation in Zebrafish in Response to the Gut

723 Microbiota. Cell Host Microbe 2: 371-382.

724 23. van der Sar, A. M., O. W. Stockhammer, C. van der Laan, H. P. Spaink, W. Bitter, and A. H.

725 Meijer. 2006. MyD88 innate immune function in a zebrafish embryo infection model. Infect.

726 Immun. 74: 2436-41.

727 24. Forn-Cuní, G., M. Varela, P. Pereiro, B. Novoa, and A. Figueras. 2017. Conserved gene 
regulation during acute inflammation between zebrafish and mammals. Sci. Rep. 7: 41905.

730 gilthead seabream professional phagocytes by different PAMPs underlines the behavioural

731 diversity of the main innate immune cells of bony fish. Mol. Immunol. 44: 2009-2016.

26. Ellett, F., L. Pase, J. W. Hayman, A. Andrianopoulos, and G. J. Lieschke. 2011. mpeg1

733 promoter transgenes direct macrophage-lineage expression in zebrafish. Blood 117: e49-e56.

734 27. Vaure, C., and Y. Liu. 2014. A Comparative Review of Toll-Like Receptor 4 Expression and

735 Functionality in Different Animal Species. Front. Immunol. 5: 316.

736 28. Lu, Y.-C., W.-C. Yeh, and P. S. Ohashi. 2008. LPS/TLR4 signal transduction pathway.

737 Cytokine 42: 145-151.

739 Innate Recognition of Lipopolysaccharide. Scand. J. Infect. Dis. 35: 568-572.

740 30. Qiao, L., W. Yang, J. Fu, and Z. Song. 2013. Transcriptome Profile of the Green Odorous

741 Frog (Odorrana margaretae). PLoS One 8: e75211.

742 31. Matsunami, M., J. Kitano, O. Kishida, H. Michimae, T. Miura, and K. Nishimura. 2015.

743 Transcriptome analysis of predator- and prey-induced phenotypic plasticity in the Hokkaido

744 salamander ( Hynobius retardatus ). Mol. Ecol. 24: 3064-3076.

745 32. Nourisson, C., M. Carneiro, M. Vallinoto, and F. Sequeira. 2014. De novo transcriptome

746 assembly and polymorphism detection in ecologically important widely distributed Neotropical

747 toads from the Rhinella marina species complex (Anura: Bufonidade. Genomic Resour. Notes

748 Accept. 1 August 2014-30 Sept. 2014. 
33. Genomic Resources Development Consortium, W. Arthofer, B. L. Banbury, M. Carneiro, F.

750 Cicconardi, T. F. Duda, R. B. Harris, D. S. Kang, A. D. Leaché, V. Nolte, C. Nourisson, N.

751 Palmieri, B. C. Schlick-Steiner, C. Schlötterer, F. Sequeira, C. Sim, F. M. Steiner, M. Vallinoto,

752 and D. A. Weese. 2015. Genomic Resources Notes Accepted 1 August 2014-30 September 2014.

753 Mol. Ecol. Resour. 15: 228-229.

754 34. Liu, Y., B. Schmidt, and D. L. Maskell. 2010. MSAProbs: multiple sequence alignment

755 based on pair hidden Markov models and partition function posterior probabilities.

756 Bioinformatics 26: 1958-1964.

757 35. Tamura, K., G. Stecher, D. Peterson, A. Filipski, and S. Kumar. 2013. MEGA6: Molecular

758 Evolutionary Genetics Analysis Version 6.0. Mol. Biol. Evol. 30: 2725-2729.

759 36. Guindon, S., J. F. Dufayard, V. Lefort, M. Anisimova, W. Hordijk, and O. Gascuel. 2010.

760 New Algorithms and Methods to Estimate Maximum-Likelihood Phylogenies: Assessing the

761 Performance of PhyML 3.0. Syst. Biol. 59: 307-321.

762 37. Le, S. Q., and O. Gascuel. 2010. Accounting for Solvent Accessibility and Secondary

763 Structure in Protein Phylogenetics Is Clearly Beneficial. Syst. Biol. 59: 277-287.

764 38. Le, S. Q., and O. Gascuel. 2008. An Improved General Amino Acid Replacement Matrix.

765 Mol. Biol. Evol. 25: 1307-1320.

766 39. Hordijk, W., and O. Gascuel. 2005. Improving the efficiency of SPR moves in phylogenetic

767 tree search methods based on maximum likelihood. Bioinformatics 21: 4338-4347.

768 40. Anisimova, M., and O. Gascuel. 2006. Approximate Likelihood-Ratio Test for Branches: A

769 Fast, Accurate, and Powerful Alternative. Syst. Biol. 55: 539-552. 
771 Principle. In Springer New York. 199-213.

772 42. Waterhouse, A. M., J. B. Procter, D. M. A. Martin, M. Clamp, and G. J. Barton. 2009.

773 Jalview Version 2-A multiple sequence alignment editor and analysis workbench. Bioinformatics

774 25: 1189-1191.

775 43. Herrero, J., M. Muffato, K. Beal, S. Fitzgerald, L. Gordon, M. Pignatelli, A. J. Vilella, S. M.

776 J. Searle, R. Amode, S. Brent, W. Spooner, E. Kulesha, A. Yates, and P. Flicek. 2016. Ensemb1

777 comparative genomics resources. Database 2016: bav096.

778 44. Farnsworth, D. R., L. Saunders, and A. C. Miller. 2019. A Single-Cell Transcriptome Atlas

779 for Zebrafish Development. bioRxiv 738344.

780 45. Zheng, G. X. Y., J. M. Terry, P. Belgrader, P. Ryvkin, Z. W. Bent, R. Wilson, S. B. Ziraldo,

781 T. D. Wheeler, G. P. McDermott, J. Zhu, M. T. Gregory, J. Shuga, L. Montesclaros, J. G.

782 Underwood, D. A. Masquelier, S. Y. Nishimura, M. Schnall-Levin, P. W. Wyatt, C. M. Hindson,

783 R. Bharadwaj, A. Wong, K. D. Ness, L. W. Beppu, H. J. Deeg, C. McFarland, K. R. Loeb, W. J.

784 Valente, N. G. Ericson, E. A. Stevens, J. P. Radich, T. S. Mikkelsen, B. J. Hindson, and J. H.

785 Bielas. 2017. Massively parallel digital transcriptional profiling of single cells. Nat. Commun. 8:

78614049.

787 46. Satija, R., J. A. Farrell, D. Gennert, A. F. Schier, and A. Regev. 2015. Spatial reconstruction 788 of single-cell gene expression data. Nat. Biotechnol. 33: 495-502.

789 47. R Core Team. 2017. R: A Language and Environment for Statistical Computing. .

790 48. Jao, L.-E., S. R. Wente, and W. Chen. 2013. Efficient multiplex biallelic zebrafish genome 
791 editing using a CRISPR nuclease system. Proc. Natl. Acad. Sci. U. S. A. 110: 13904-9.

792 49. Sun, Y., Y. Huang, X. Li, C. C. Baldwin, Z. Zhou, Z. Yan, K. A. Crandall, Y. Zhang, X.

793 Zhao, M. Wang, A. Wong, C. Fang, X. Zhang, H. Huang, J. V. Lopez, K. Kilfoyle, Y. Zhang, G.

794 Ortí, B. Venkatesh, and Q. Shi. 2016. Fish-T1K (Transcriptomes of 1,000 Fishes) Project: Large-

795 scale transcriptome data for fish evolution studies. Gigascience 5.

796 50. Woods, I. G., C. Wilson, B. Friedlander, P. Chang, D. K. Reyes, R. Nix, P. D. Kelly, F. Chu,

797 J. H. Postlethwait, and W. S. Talbot. 2005. The zebrafish gene map defines ancestral vertebrate

798 chromosomes. Genome Res. 15: 1307-14.

799 51. Bernut, A., J.-L. Herrmann, K. Kissa, J.-F. Dubremetz, J.-L. Gaillard, G. Lutfalla, and L.

800 Kremer. 2014. Mycobacterium abscessus cording prevents phagocytosis and promotes abscess

801 formation. Proc. Natl. Acad. Sci. 111: E943-E952.

802 52. Heumann, D., R. Lauener, and B. Ryffel. 2003. The dual role of LBP and CD14 in response

803 to Gram-negative bacteria or Gram-negative compounds. J. Endotoxin Res. 9: 381-384.

804 53. Triantafilou, M., and K. Triantafilou. 2002. Lipopolysaccharide recognition: CD14, TLRs

805 and the LPS-activation cluster. Trends Immunol. 23: 301-304.

806 54. Kim, S. J., and H. M. Kim. 2017. Dynamic lipopolysaccharide transfer cascade to

807 TLR4/MD2 complex via LBP and CD14. BMB Rep. 50: 55-57.

808 55. Shapiro, R. A., M. D. Cunningham, K. Ratcliffe, C. Seachord, J. Blake, J. Bajorath, A.

809 Aruffo, and R. P. Darveau. 1997. Identification of CD14 residues involved in specific

810 lipopolysaccharide recognition. Infect. Immun. 65: 293-7.

811 56. Juan, T. S.-C., E. Hailman, M. J. Kelley, S. D. Wright, and H. S. Lichenstein. 1995. 
812 Identification of a Domain in Soluble CD14 Essential for Lipopolysaccharide (LPS) Signaling

813 but Not LPS Binding. J. Biol. Chem. 270: 17237-17242.

814 57. Zhou, Y., Q. Liang, W. Li, Y. Gu, X. Liao, W. Fang, and X. Li. 2016. Characterization and

815 functional analysis of toll-like receptor 4 in Chinese soft-shelled turtle Pelodiscus sinensis. Dev.

816 Comp. Immunol. 63: 128-135.

817 58. Madan, R., D. Golenbock, S. Divanovic, A. Trompette, S. F. Atabani, D. T. Golenbock, A.

818 Visintin, R. W. Finberg, A. Tarakhovsky, S. N. Vogel, Y. Belkaid, E. A. Kurt-Jones, and C. L.

819 Karp. 2005. Negative Regulation of TLR4 Signaling by RP105. .

820 59. Hughes, L. C., G. Ortí, Y. Huang, Y. Sun, C. C. Baldwin, A. W. Thompson, D. Arcila, R.

821 Betancur-R., C. Li, L. Becker, N. Bellora, X. Zhao, X. Li, M. Wang, C. Fang, B. Xie, Z. Zhou,

822 H. Huang, S. Chen, B. Venkatesh, and Q. Shi. 2018. Comprehensive phylogeny of ray-finned

823 fishes (Actinopterygii) based on transcriptomic and genomic data. Proc. Natl. Acad. Sci. 115:

$824 \quad 6249-6254$.

825 60. Hedges, S. B., J. Dudley, and S. Kumar. 2006. TimeTree: a public knowledge-base of

826 divergence times among organisms. Bioinformatics 22: 2971-2972.

827 61. Muroi, M., T. Ohnishi, and K.-I. Tanamoto. 2002. MD-2, a Novel Accessory Molecule, Is

828 Involved in Species-Specific Actions of Salmonella Lipid A. Infect. Immun. 70: 3546-3550.

829 62. Hoshino, K., O. Takeuchi, T. Kawai, H. Sanjo, T. Ogawa, Y. Takeda, K. Takeda, and S.

830 Akira. 1999. Cutting edge: Toll-like receptor 4 (TLR4)-deficient mice are hyporesponsive to

831 lipopolysaccharide: evidence for TLR4 as the Lps gene product. J. Immunol. 162: 3749-52.

832 63. Yang, D., X. Zheng, S. Chen, Z. Wang, W. Xu, J. Tan, T. Hu, M. Hou, W. Wang, Z. Gu, Q. 
833 Wang, R. Zhang, Y. Zhang, and Q. Liu. 2018. Sensing of cytosolic LPS through caspy2 pyrin

834 domain mediates noncanonical inflammasome activation in zebrafish. Nat. Commun. 9: 3052.

835 64. Forn-Cuní, G., A. H. Meijer, and M. Varela. 2019. Zebrafish in Inflammasome Research.

836 Cells 8: 901.

837 65. Mazon-Moya, M. J., A. R. Willis, V. Torraca, L. Boucontet, A. R. Shenoy, E. Colucci-

838 Guyon, and S. Mostowy. 2017. Septins restrict inflammation and protect zebrafish larvae from

839 Shigella infection. PLOS Pathog. 13: e1006467.

840 66. Jones, J. D. G., R. E. Vance, and J. L. Dangl. 2016. Intracellular innate immune surveillance

841 devices in plants and animals. Science (80-. ). 354: aaf6395-aaf6395.

842 67. Bernheiden, M., J.-M. Heinrich, G. Minigo, C. Schutt, F. Stelter, M. Freeman, D. Golenbock, 843 and R. S. Jack. 2001. LBP, CD14, TLR4 and the murine innate immune response to a peritoneal

844 Salmonella infection. J. Endotoxin Res. 7: 447-450.

845 68. Loes, A. N., J. T. Bridgham, and M. J. Harms. 2018. Coevolution of the Toll-Like Receptor 4

846 Complex with Calgranulins and Lipopolysaccharide. Front. Immunol. 9: 304.

847 69. Ehrchen, J. M., C. Sunderkotter, D. Foell, T. Vogl, and J. Roth. 2009. The endogenous Toll-

848 like receptor 4 agonist S100A8/S100A9 (calprotectin) as innate amplifier of infection,

849 autoimmunity, and cancer. J. Leukoc. Biol. 86: 557-566.

850 70. Ohto, U., K. Fukase, K. Miyake, and T. Shimizu. 2012. Structural basis of species-specific

851 endotoxin sensing by innate immune receptor TLR4/MD-2. Proc. Natl. Acad. Sci. 109: 7421-

8527426.

853 71. Walsh, C., M. Gangloff, T. Monie, T. Smyth, B. Wei, T. J. McKinley, D. Maskell, N. Gay, 
854 and C. Bryant. 2008. Elucidation of the MD-2/TLR4 Interface Required for Signaling by Lipid

855 IVa. J. Immunol. 181: 1245-1254.

856 72. Anderson, J. A., A. N. Loes, G. L. Waddell, and M. J. Harms. 2019. Tracing the evolution of

857 novel features of human Toll-like receptor 4. Protein Sci. 28: pro.3644.

858 73. Iliev, D. B., J. C. Roach, S. Mackenzie, J. V Planas, and F. W. Goetz. 2005. Endotoxin

859 recognition: in fish or not in fish? FEBS Lett. 579: 6519-28.

860 74. Berczi, I., L. Bertók, and T. Bereznai. 1966. Comparative studies on the toxicity of

861 Escherichia coli lipopolysaccaride endotoxin in various animal species. Can. J. Microbiol. 12:

$862 \quad 1070-1071$.

863 75. Stafford, J. L., K. K. Ellestad, K. E. Magor, M. Belosevic, and B. G. Magor. 2003. A toll-like

864 receptor (TLR) gene that is up-regulated in activated goldfish macrophages. Dev. Comp.

865 Immunol. 27: 685-698.

866 76. MacKenzie, S., J. V. Planas, and F. W. Goetz. 2003. LPS-stimulated expression of a tumor

867 necrosis factor-alpha mRNA in primary trout monocytes and in vitro differentiated macrophages.

868 Dev. Comp. Immunol. 27: 393-400.

869 77. Pelegrín, P., J. García-Castillo, V. Mulero, and J. Meseguer. 2001. Interleukin-1 $\beta$ isolated

870 from a marine fish up-regulated expression in macrophages following activation with

871 lipopolysaccharide and lymphokines. Cytokine 16: 67-72.

872 78. Ishii, A., M. Kawasaki, M. Matsumoto, S. Tochinai, and T. Seya. 2007. Phylogenetic and

873 expression analysis of amphibian Xenopus Toll-like receptors. Immunogenetics 59: 281-293. 